\title{
Serum lysyl oxidase concentration increases in long-standing systemic sclerosis: Can lysyl oxidase change over time?
}

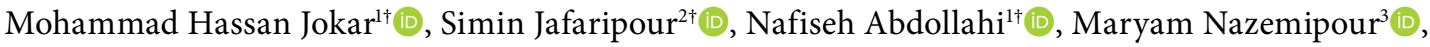 \\ Maliheh Moradzadeh ${ }^{1}(\mathbb{D})$, Mohammad Ali Mansournia ${ }^{4+}(\mathbb{C}$ \\ ${ }^{1}$ Golestan Rheumatology Research Center, Sayad Shirazi Hospital, Golestan University of Medical Sciences, Gorgan, Iran \\ ${ }^{2}$ Department of Medical Genetics, School of Medicine, Mashhad University of Medical Sciences, Mashhad, Iran \\ ${ }^{3}$ Psychosocial Health Research Institute, Iran University of Medical Sciences, Tehran, Iran \\ ${ }^{4}$ Department of Epidemiology and Biostatistics, School of Public Health, Tehran University of Medical Sciences, Tehran, Iran
}

\begin{abstract}
Objectives: This study aims to investigate the association of serum lysyl oxidase (LOX) levels with systemic sclerosis (SSc), to examine the relationship between LOX and disease onset, and to evaluate the probable effects of hyperlipidemia on the circulating levels of LOX among patients with SSC.

Patients and methods: Between May 2017 and November 2018, a total of 39 patients with SSc ( 2 males, 37 females; mean age: $46.6 \pm 12.3$ years; range, 18 to 65 years) and 35 healthy controls ( 4 males, 31 females; mean age: $43.1 \pm 14.1$ years; range, 18 to 65 years) were included. Serum LOX concentration was measured using the enzyme-linked immunoassay in triplicate.

Results: We found higher levels of serum LOX in patients with SSc compared to healthy controls. There was a significant relationship between serum LOX levels and disease onset. Patients with long-standing disease demonstrated increased levels of LOX in the blood compared to the recent-onset group. Hyperlipidemia did not have a significant effect on circulating levels of LOX. There was a significant negative correlation between LOX levels and modified Rodnan Skin Score in the subgroup of patients with skin involvement only and in patients without gastrointestinal involvement.

Conclusion: Our study findings show an increased level of LOX protein level in the blood of patients diagnosed with SSc. Hyperlipidemia seems not to affect the concentrations of LOX in the peripheral blood of patients with SSc.

Keywords: Biomarker, hyperlipidemia, long-standing disease, lysyl oxidase, systemic sclerosis.
\end{abstract}

The lysyl oxidase (LOX) gene is placed on the long arm of chromosome 5 and encodes the most studied member of the proteins belonging to the LOX family, protein-lysine 6-oxidase, commonly known as LOX $^{1}$ which is a copper-dependent extracellular enzyme. ${ }^{2}$ The most well-known biological function of the LOX family is their oxidative deamination of lysine and hydroxyl lysine residues in both collagen and elastin molecules, the major components of the extracellular matrix (ECM). ${ }^{3}$ This activity leads to the biosynthesis of normal covalently linked collagen and elastin fibers, thus providing the structural integrity of ECM and, accordingly, connective tissues. ${ }^{3}$ The other main functions of the LOX family include playing active roles in processes such

Received: May 30, 2021 Accepted: August 02, 2021 Published online: October 18, 2021

Correspondence: Maliheh Moradzadeh, PhD. Golestan Rheumatology Research Center, Sayad Shirazi Hospital, Golestan University of Medical Sciences, 4917867439 Gorgan, Iran. Tel: 9112791382 e-mail: moradzadeh63@yahoo.com

† These authors contributed equally to this paper.

\section{Citation:}

Jokar MH, Jafaripour S, Abdollahi N, Nazemipour M, Moradzadeh M, Mansournia MA. Serum lysyl oxidase concentration increases in long-standing systemic sclerosis: Can lysyl oxidase change over time?. Arch Rheumatol 2022;37(2):261-270. 
as cell differentiation, ${ }^{4}$ cell proliferation, ${ }^{5}$ cell migration, ${ }^{6}$ and angiogenesis. ${ }^{7}$ Unsurprisingly, there is clear evidence suggesting that abnormal changes in either LOX gene expression levels or LOX protein activity occur in various pathological conditions in humans, from cancer disorders to aortic aneurysms. ${ }^{8}$ Among them, fibrotic diseases are one of the earliest-reported groups shown to be associated with elevated levels of LOX in the involved tissues and organs. ${ }^{9}$ Indeed, LOX expression levels increase in the lungs of individuals suffering from idiopathic pulmonary arterial hypertension, ${ }^{10}$ patients with heart and liver fibrosis, and dermal fibroblast cells from those with systemic sclerosis (SSc). ${ }^{11}$

In SSc, as a rare inflammatory disease of the connective tissue, fibrotic changes accompanying elevated LOX levels can occur in both the skin and lung. ${ }^{12}$ Nishimoto et al. ${ }^{13}$ reported an increased level of LOX protein in the lung fibroblasts of patients with SSc. Interestingly, enhanced levels of LOX have been demonstrated not only in the skin and lung fibroblasts, but also in the peripheral blood of individuals with SSc. ${ }^{14,15}$ This recent observation first came from a study on 26 patients with SSc and 25 healthy controls conducted by Rimar et al. ${ }^{15}$ The team, then, validated their preliminary finding in a study with a larger sample size. ${ }^{14}$ They also suggested the lung as a possible source of LOX protein in the sera of patients with $\mathrm{SSc}$ and proposed circulating levels of LOX as a potential biomarker in SSc. Undoubtedly, biomarkers are of great importance in the assessment of patients in terms of disease status, disease severity, and response to drug therapy. ${ }^{16,17}$ In particular, in $\mathrm{SSc}$, novel biomarkers are in great demand due to the yet unknown major underlying cause and since non-selective therapeutic options are available to all patients, regardless of the disease progression level. ${ }^{19}$ Taking the life-threatening nature of SSc into consideration, scientists demand to use novel biomarkers as tools for either the prediction of disease progression or regression or as candidate surrogate measure in early phases of drug discovery. ${ }^{19}$ To be considered as a disease status or severity biomarker, the candidate maker must contain two essential features: a strong correlation with some widely accepted clinical outcome, and the ability to undergo significant changes over time. ${ }^{20}$
In case of circulating levels of LOX, the literature has shown its association with a diffusing capacity of the lungs for carbon monoxide (DLCO) as an indication of pulmonary fibrosis. ${ }^{18}$ On the other hand, the ability of LOX to change over time has not yet been reported, although it has been correlated relatively well with SSc severity $(r=0.26, p=0.017) .{ }^{17}$ In terms of sampling, considering blood as a source of LOX measure has a great advantage of convenient and repeatable measurement, although it makes LOX levels susceptible to being affected by the vascular source of the protein and its associated factors. Indeed, atherogenic serum concentrations of low-density lipoprotein (LDL) are shown to be associated with decreased LOX levels in the aortic cells from porcine animal models. ${ }^{21}$ Decreased and increased LOX expression levels have been reported in the early stages and advanced levels of atherosclerosis, respectively. ${ }^{21}$ Therefore, it is not unlikely that increased levels of LDL and dyslipidemia should affect LOX protein levels in the blood.

In the present study, we primarily aimed to validate circulating levels of LOX as a biomarker of disease status in SSc. We, therefore, investigated (i) the association of LOX with $\mathrm{SSc}$ and (ii) whether there was any relationship between LOX and disease onset (recent-onset versus long-standing disease), i.e., whether LOX could change over time. Taking into account hyperlipidemia as a potential confounding factor, we also aimed to explore the relationship of hyperlipidemia with circulating levels of LOX among patients with SSc to eliminate any possible effect of hyperlipidemia on the assessment of the relationship between LOX and SSc.

\section{PATIENTS AND METHODS}

This cross-sectional study was conducted at rheumatology clinic of Sayad Shirazi Educational and Medical Center, Gorgan, Iran between May 2017 and November 2018. A total of 39 consecutive patients with SSc (2 males, 37 females; mean age: $46.6 \pm 12.3$ years; range, 18 to 65 years) who were diagnosed by an experienced rheumatologist and 35 healthy controls (4 males, 31 females; mean age: $43.1 \pm 14.1$ years; range, 18 to 65 years) were 
included. Patients aged $<18$ years, having a positive pregnancy test, a positive history of any active infection during the past six months, or having diagnosed with any other autoimmune disease such as fibromyalgia syndrome, rheumatoid arthritis, myasthenia gravis, and diabetes mellitus were excluded. Demographic characteristics including sex, age, and marital status and clinical data including a history of hyperlipidemia were obtained by an interviewer or collected from the clinical records of the patients.

Hyperlipidemia was defined either based on the patients' self-report that was consistent with patient's clinical history or their current use of lipid-lowering medicine. Clinical data on $\mathrm{SSc}$, including the subtype of SSc, the modified Rodnan Skin Score (mRSS), gastrointestinal (GI) tract and cardiac involvement, and lung fibrosis were assessed by a rheumatologist during visits and/or based on the results of radiographic examinations and laboratory tests. All patients underwent medical examination for the identification of SSc subtype as either diffuse or limited based on the criteria established by the American College of Rheumatology $(\mathrm{ACR})^{22}$ and for the evaluation of mRSS according to skin thickness assessment. ${ }^{23}$ The patients were also categorized based on the disease duration following diagnosis into two groups: recentonset (disease duration $\leq 5$ years) and longstanding disease (disease duration $>5$ years). Disease onset was considered the development of Raynaud's phenomenon as the earliest clinical symptom of SSc. To determine the development and/or the extent of GI tract manifestations, data regarding reflux, abdominal distension, early satiety, vomiting, diarrhea, fecal incontinence, and constipation were collected according to a checklist. In addition, all the patients underwent a barium swallow test and computed tomography (CT) enterography procedure. Cardiac involvement was defined as the presence of indications of myocardial ischemia, severe arrhythmias and/or atrioventricular conduction abnormalities, pericarditis, and congestive heart failure (all assessed based on clinical symptoms, electrocardiogram [ECG], and echocardiography). Furthermore, lung fibrosis was diagnosed if $>20 \%$ of the lung demonstrated fibrotic changes on high-resolution CT scan. The analysis of micronutrients, including vitamin B12, folic acid, zinc $(\mathrm{Zn})$, magnesium $(\mathrm{Mg})$, and albumin was also performed to investigate the nutritional status and any deficiency in the studied factors. The controls were healthy individuals who visited our center for annual medical examinations and showed no symptom of any particular autoimmune illness.

\section{Laboratory measurements}

A blood sample was taken from each patient and transferred to the laboratory for examination. The erythrocyte sedimentation rate (ESR) was measured by an automated ESR analyzer (Electa Co., NJ, USA) using the Westergren ESR method and reported as $\mathrm{mm} / \mathrm{h}$. Ethylenediaminetetraacetic acid (EDTA)-containing blood samples were also utilized for the complete blood count (CBC) test via a cell counter (Nihon Kohden Celltac E, Nihon Kohden Corporation, Tokyo, Japan). Commercially available enzyme-linked immunoassay (ELISA) kits were employed for the detection of anti-centromere antibody (ACA) and autoantibodies against topoisomerase I (anti-Scl-70 antibody) in sera and for the evaluation of serum LOX concentrations in all the participants (MyBioSource, CA, USA) according to the manufacturer's instruction. For each sample, the values for LOX concentration were re-assayed in triplicate and reported as $\mathrm{ng} / \mathrm{mL}$. Air-acetylene flame atomic absorption spectroscopy was adopted to determine $\mathrm{Zn}$ using a spectrometer (Varian Inc., CA, USA). The levels of $\mathrm{Mg}$ and albumin were also measured using commercially available kits (Pars Azmun Inc., Tehran, Iran). Finally, folic acid and vitamin B12 levels were simultaneously measured using a radioimmunoassay kit (MP Biomedicals Inc., CA, USA).

\section{Cut-off values}

Regarding autoantibodies, the positive cutoff values for the test were considered as serum concentrations $>25 \mathrm{EU} / \mathrm{mL}$ and $>20 \mathrm{EU} / \mathrm{mL}$ for ACA and anti-Scl-70 antibody, respectively. Vitamin B12, folic acid, Zn, and Mg deficiency were defined as the concentrations of $<200 \mathrm{pg} / \mathrm{mL}$, $<2.2 \mathrm{ng} / \mathrm{mL},<70 \mathrm{mg} / \mathrm{dL}$, and $<1.5 \mathrm{mmol} / \mathrm{L}$, respectively. Low plasma albumin was defined as an albumin level of $<3.5 \mathrm{~g} / \mathrm{dL}$. 


\section{Statistical analysis}

Statistical analysis was performed using the SPSS for Windows version 11.5 (SPSS Inc., Chicago, IL, USA) and STATA version 15.0 software (StataCorp LLC, College Station, TX, USA). Continuous data were expressed in mean \pm standard deviation (SD) or median (min-max), while categorical data were expressed in number and frequency. Disease subtype categories were compared using two-tailed t-tests in the case of continuous variables where conditions for the parametric test were met, and the Mann-Whitney U test was employed for data which did not satisfy the assumptions of the parametric test. Categories were compared using the chi-square test in the case of categorical variables. The relationship between LOX levels and variables of interest (group, disease type, lung fibrosis, cardiac and GI involvement, onset, and hyperlipidemia) was assessed using regression analysis, and the regression coefficient, the statistical significance of the regression model, and the proportion of
LOX (as the dependent variable) determined by these variables were computed. If needed, to normalize the data, the analysis was performed using log-transformed data, and to calculate the confidence interval, the bootstrapping method was employed. The correlation between LOX levels and disease-related covariates was also assessed. The Pearson correlation analysis was employed to calculate the correlation coefficient (R) of LOX concentration. A two-tailed $p$ value of $<0.05$ was considered statistically significant.

\section{RESULTS}

Of 39 patients, 22 had diffuse SSc and 17 had limited SSc. Also, 10 patients were categorized into recent-onset and 29 patients into long-standing disease groups. There were no significant differences between case and control groups in terms of age and sex. There was no statistically significant difference between the two subtypes of $\mathrm{SSc}$, diffuse and limited,

Table 1. Demographic and clinical characteristics of the participants

\begin{tabular}{|c|c|c|c|c|c|c|c|c|c|c|c|}
\hline & \multicolumn{6}{|c|}{ Patients with SSc $(\mathrm{n}=39)$} & \multicolumn{3}{|c|}{ Healthy controls $(\mathrm{n}=35$ ) } & \multirow{3}{*}{$\begin{array}{c}\text { Patients vs. } \\
\text { controls } \\
p\end{array}$} & \multirow{3}{*}{$\begin{array}{c}\text { Diffuse SSc vs } \\
\text { Limited SSc } \\
p\end{array}$} \\
\hline & \multicolumn{3}{|c|}{ Diffuse SSc $(n=22)$} & \multicolumn{3}{|c|}{ Limited SSc (n=17) } & \multirow[b]{2}{*}{$\mathrm{n}$} & \multirow[b]{2}{*}{$\%$} & \multirow[b]{2}{*}{ Mean $\pm S D$} & & \\
\hline & $\mathrm{n}$ & $\%$ & Mean \pm SD & $\mathrm{n}$ & $\%$ & Mean \pm SD & & & & & \\
\hline Age (year) & & & $44.9 \pm 11.4$ & & & $47.3 \pm 13.4$ & & & $43.11 \pm 14.06$ & 0.2 & 0.54 \\
\hline Sex & & & & & & & & & & & \\
\hline Male & 1 & 4.5 & & 1 & 5.9 & & 4 & 11.42 & & 0.32 & 0.85 \\
\hline Years since diagnosis & & & $10.0 \pm 7.6$ & & & $14.2 \pm 8.0$ & & & & - & 0.11 \\
\hline mRSS & & & $17 \pm 9.1$ & & & $12.7 \pm 7.9$ & & & & & 0.16 \\
\hline Lung fibrosis & 4 & 18.2 & & 7 & 41.2 & & & & & & 0.1 \\
\hline GI involvement & 14 & 63.6 & & 13 & 76.5 & & & & & & 0.38 \\
\hline Cardiac involvement & 6 & 27.3 & & 2 & 11.8 & & & & & & 0.23 \\
\hline Hyperlipidemia & 7 & 31.8 & & 5 & 29.4 & & & & & & 0.87 \\
\hline Diarrhea & 6 & 27.3 & & 5 & 29.4 & & & & & & 0.8 \\
\hline ACA & 2 & 9.1 & & 6 & 35.3 & & & & & & 0.04 \\
\hline Anti SLC70 & 15 & 68.2 & & 3 & 17.6 & & & & & & 0.002 \\
\hline ESR & & & $38.9 \pm 23.7$ & & & $35.1 \pm 25.0$ & & & & & 0.63 \\
\hline Low plasma albumin & 3 & 13.6 & & 4 & 25 & & & & & & 0.37 \\
\hline Folic acid deficiency & 0 & 0 & & 1 & 6.3 & & & & & & 0.23 \\
\hline Vitamin B12 deficiency & 2 & 9.1 & & 0 & 0 & & & & & & 0.21 \\
\hline Zn deficiency & 20 & 90.9 & & 15 & 93.8 & & & & & & 0.75 \\
\hline
\end{tabular}


regarding any variable of interest other than ACA and anti-Scl-70. The frequency of ACA-positive participants was higher among patients with limited SSc $(35.3 \%$ vs. 9.1\%, respectively; $p=0.04$ ), while the number of anti-Scl-70-positive patients was higher among those with the diffuse subtype (68.2 vs. 17.6, respectively; $p=0.002)$. Table 1 shows demographic and disease-related characteristics of the participants, as well as the results of the comparison between diffuse and limited SSc subgroups regarding all the investigated variables except for $\mathrm{Mg}$ deficiency, as ecause the entire sample had normal values of $\mathrm{Mg}$ in their serum at the time of the experiment (range, 1.5 to $2.5 \mathrm{mmol} / \mathrm{L}$ ).

Figure 1 and 2 represent scatter/dot plot of the LOX levels in patients versus healthy controls and in patients with limited cutaneous SSc versus diffuse cutaneous SSc, respectively. Table 2 represents the results of the regression analysis of LOX levels with the following variables: group, disease type, onset, internal organ involvement (GI and cardiac involvement), hyperlipidemia, and lung fibrosis. Table 3 presents the results of the regression analysis of LOX with onset in patients with diffuse and limited types of SSc.

The relationship between serum concentrations of LOX protein levels as well as log-transformed values of LOX and group variable (case versus control) was not statistically significant in the full sample of

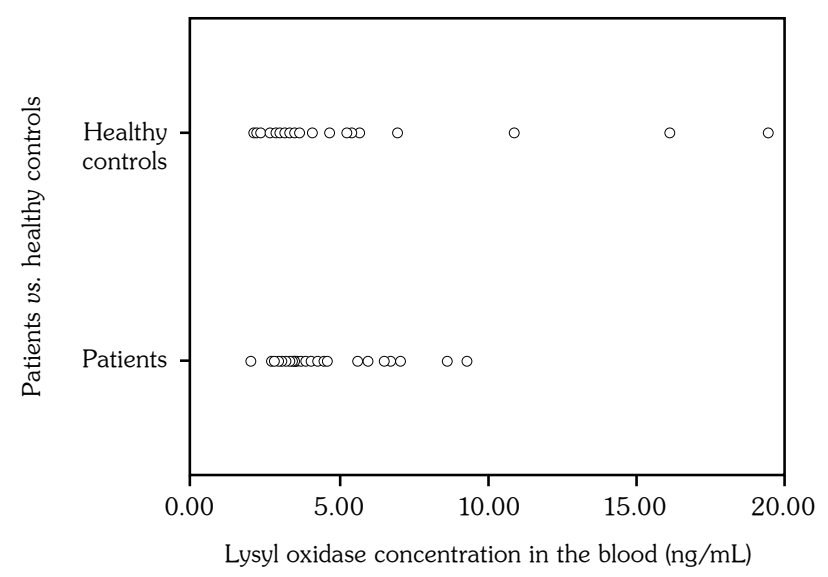

Figure 1. LOX concentration in the blood among patients versus controls.

LOX: Lysyl oxidase. the study (Table 2). However, when only the participants with LOX levels of $<16 \mathrm{ng} / \mathrm{mL}$ were included in the analysis, the regression established that group could significantly predict the log-transformed values of LOX $(p=0.047$, $r=-0.132,95 \%$ confidence interval $[\mathrm{CI}]:-0.26$, -0.002 ) and account for $2.5 \%$ (adjusted $\mathrm{R}^{2}$ ) of LOX variability (data not shown in the table). Moreover, natural logarithm of LOX values were 0.13 higher among patients with SSc compared to healthy controls. Table 2 also shows that among the investigated variables, only onset could significantly affect LOX $(p=0.019)$ and account for $2 \%$ of the explained variability in serum LOX concentrations in patients with SSc. Indeed, LOX levels in patients with long-standing disease were 0.78 higher compared to patients with recent-onset SSc. Furthermore, the effect of onset was significant $(p=0.047)$ in patients with the diffuse type of $\mathrm{SSc}$, yet such a significant effect was not observed in patients with limited SSc (Table 3). On the other hand, the interaction between onset and disease type was not statistically significant $(p=0.45)$. As for disease type, lung fibrosis, cardiac and GI involvement, and hyperlipidemia, their effects on LOX circulating levels were all statistically insignificant.

Zinc deficiency was very common among the patients (92.1\%), whereas vitamin B12 and folic acid deficiencies were less common $(5.3 \%$ and $2.6 \%$, respectively). Furthermore, no case with

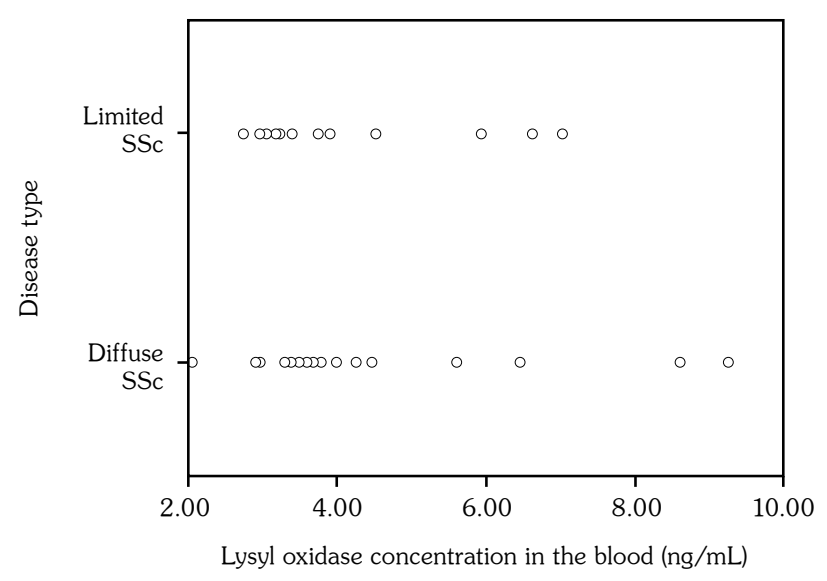

Figure 2. LOX concentration in the blood in patients with limited versus diffuse type of SSc.

LOX: Lysyl oxidase; SSc: Systemic sclerosis. 


\begin{tabular}{|c|c|c|c|c|c|}
\hline \multirow[b]{2}{*}{ Sample } & \multirow[b]{2}{*}{ Predictor } & \multirow[b]{2}{*}{$p$} & \multirow[b]{2}{*}{ Coefficient } & \multicolumn{2}{|c|}{$95 \% \mathrm{CI}$} \\
\hline & & & & Lower bound & Upper bound \\
\hline \multirow[t]{3}{*}{ Full sample } & Group (case vs. control) & 0.67 & -0.038 & -0.22 & 0.14 \\
\hline & Disease subtype & 0.91 & -0.049 & -0.92 & 0.82 \\
\hline & Lung fibrosis & 0.89 & 0.098 & -1.28 & 1.48 \\
\hline \multirow[t]{3}{*}{ Patients } & Cardiac \& gastrointestinal involvement & 0.83 & -0.09 & -0.91 & 0.73 \\
\hline & Onset & 0.019 & 0.78 & 0.13 & 1.44 \\
\hline & Hyperlipidemia & 0.14 & 0.65 & -0.21 & 1.52 \\
\hline
\end{tabular}

\begin{tabular}{|c|c|c|c|c|c|}
\hline \multirow[b]{2}{*}{ Sample } & \multirow[b]{2}{*}{ Predictor } & \multirow[b]{2}{*}{$p$} & \multirow[b]{2}{*}{ Coefficient } & \multicolumn{2}{|c|}{$95 \% \mathrm{CI}$} \\
\hline & & & & Lower bound & Upper bound \\
\hline Patients with diffuse SSc & Onset & 0.047 & 1.01 & 0.009 & 2.01 \\
\hline Patients with limited SSc & Onset & 0.49 & 0.42 & -0.78 & 1.63 \\
\hline
\end{tabular}

Mg deficiency was found among the patients. In terms of vitamin B12, folic acid, and $\mathrm{Zn}$ deficiency, and also the percentage of individuals who had low levels of plasma albumin, there were no significant differences between the two groups of patients who had different subtypes of SSc. The means of the measured serum levels of vitamin B12, folic acid, $\mathrm{Mg}, \mathrm{Zn}$, and albumin were as follows: $435.9 \pm 182.5,14.8 \pm 7.1,2.0 \pm 0.1$, $48.1 \pm 28.8$, and $4.0 \pm 0.4$.

The correlation analysis between serum LOX levels and the investigated variables revealed no link between LOX levels and the means of age, duration of the disease, mRSS, ESR, vitamin $\mathrm{B} 12$, folic acid, $\mathrm{Mg}, \mathrm{Zn}$, and albumin. Nevertheless, LOX concentrations demonstrated a trend toward a negative correlation with albumin levels only in the subgroup of patients with diffuse SSc $(r=-0.43$, $p=0.05)$. There was also a significant negative correlation between LOX levels and mRSS in the subgroup of patients with skin involvement only $(\mathrm{r}=-0.66, \mathrm{p}=0.03)$ and also in patients without GI involvement $(r=-0.77, p=0.009)$.

\section{DISCUSSION}

Our study findings demonstrated significantly higher levels of log-transformed values of LOX in patients with SSc compared to the healthy controls among participants with LOX $<16 \mathrm{ng} / \mathrm{mL}$. Among this group, the mean levels of LOX were slightly higher among patients compared to the healthy controls $(4.14 \pm 1.64$ vs. $3.64 \pm 1.66 \mathrm{ng} / \mathrm{mL}$ ). Moreover, at the time of measurement, the long-standing SSc participants had significantly higher levels of LOX than the recent-onset group. A significant relationship between LOX and onset was also found in patients with the diffuse subtype of the disease, but not in patients diagnosed with the limited subtype. In addition, LOX was not associated with any particular type of disease, any internal organ involvement, or hyperlipidemia. The correlation analysis also revealed a negative correlation between LOX levels and mRSS solely among patients who were not diagnosed with GI tract involvement. The results also demonstrated that the majority of the patients had $\mathrm{Zn}$ deficiency. 
It has been well established that the enzymatic function of LOX is vital for the development of the mature three-dimensional structure of ECM. ${ }^{3}$ The LOX can also contribute to the regulation of the production levels of other ECM components such as collagen type III. ${ }^{24}$ As mentioned earlier, tissue-specific elevated levels of LOX are associated with fibrotic changes. ${ }^{11}$ It was about five years ago, when LOX was suggested as a potential biomarker in SSc. ${ }^{14}$ To the best of our knowledge, this is the first independent study since then to re-investigate the possible relationship between serum levels of LOX and $\mathrm{SSc}$, and the first to report the association between LOX and disease onset. The assessment of serum concentration of LOX as a biomarker in $\mathrm{SSc}$ seems to be particularly interesting. Due to the challenge of obtaining biopsy samples of the internal involved organs, it would be ideal to find biomarkers for disease status or severity in the blood, as blood can be easily considered as a source of repeated measures. Evidence from the literature also demonstrated higher LOX levels in patients than in controls, and its correlation with disease severity and DLCO in diffuse SSc. ${ }^{14,18}$ In line with the literature, we found higher levels of natural logarithm of LOX in patients than in healthy controls, if only participants measured with a LOX of $<16 \mathrm{ng} / \mathrm{mL}$ were included in the analysis. Indeed, there were a few outliers in our data, more frequently in the control group, although the inclusion criteria were rigorous and the method for assessing LOX protein levels was thorough. Our results underscore the importance of defining normal ranges of LOX in human populations and its determining factors, since the factors, genes, or regulatory pathways affecting LOX protein levels have not been fully understood, yet. In addition, the mean levels of LOX gene expression in the human populations may naturally vary. Indeed, $17 \%$ of our genes, such as SH2B3, are expressed differentially in different populations, ${ }^{25}$ and some of our protein-coding genes have demonstrated a population-specific splicing ratio. ${ }^{26}$ In the present study, we also found a significant relationship between LOX protein levels and the onset of SSc; the patients with long-standing disease showed increased levels of LOX compared to the recent-onset group. Moreover, the effect of disease onset was only significant in patients with the diffuse subtype, although we could not deny the effect of onset in patients diagnosed with limited SSc. Our observed relationship between LOX and onset is in contrast to what was reported by Vadasz et al. ${ }^{14} \mathrm{~A}$ reason for these contradictory results might be that the period between the first diagnosis and enrolment in the study was on average about two times longer in our study than in the study of Vadasz et al. ${ }^{14}$ (11.84 vs. 6.7 years, respectively). However, in both studies, the patients were almost at the end stages of the disease. Probably, detectable changes in serum LOX levels only happen during longer periods of SSc. Our results imply that, as a molecule directly involved in the fibrotic process (a key part of disease pathogenesis), LOX may be able to change over time. It is hypothesized that the observed association of LOX with disease onset can be related to the progression of the disease. In view of our findings and the previous evidence for the correlation of LOX with disease severity, ${ }^{18}$ it is likely that LOX should reflect the aggravation of the disease and changes in the pathogenic process during the disease course. There may also be an association between LOX and the aggravation of the entire fibrotic process in the disease. Thus, it may be proved useful in the assessment of either disease progression or regression and response to drug therapy in clinical trials. However, since the sample size in this study was relatively small and LOX protein levels were only measured at one point in time, our results should be interpreted with caution. Further studies with larger cohorts of patients, more informative measurements of disease outcomes, and measurement of LOX levels at multiple points in time are required to validate the ability of LOX to change over time. Based on our findings, consistent with the results of Vadasz et al., ${ }^{14}$ there was no statistically significant relationship between serum LOX concentrations and pulmonary fibrosis. However, they reported strong LOX staining in the lung biopsies of patients with SSc compared to healthy controls. We found the same evidence according to the relationship between LOX and GI tract, as well as heart involvement. Also, none of these organ involvements was seemingly of substantial impact on the circulating levels of LOX. It is more likely that obtaining biopsies and applying more effective methods for determining the burden of organ fibrosis should yield better results on the relationship between serum LOX concentrations 
and internal organ fibrosis. As for the methods of determining the degree of fibrosis, a good example can be the measurement of collagen staining by Sirius Red in connective tissues. ${ }^{27} \mathrm{On}$ the other hand, serum LOX and single-tissue LOX concentrations need not be related due to differences in the respective microenvironments, rates of synthesis and degradation, and differences in the possible sources of the measured enzymes. A combination of all the involved organs and accurate assessment of the extent of the organ disease may provide an overall picture of the disease status and far greater chances to detect such a relationship. Consistent with the Vadasz et al.'s ${ }^{14}$ study, our findings also showed that LOX was not significantly correlated with mRSS. Interestingly, in their study, skin biopsies from patients with long-standing severe diffuse SSc showed poor LOX staining, relatively similar to those of healthy controls. This observation may support the hypothesis that the expression levels of LOX can widely vary among involved organs. In addition, our results driven from the correlation analysis demonstrated that, in the late stages of the disease which was either restricted to skin tissues or did not involve the GI tract, more skin involvement is correlated with lower LOX levels in the blood. A probable explanation can be that various pathogenic processes may be involved in different patterns of organ involvement in SSc. Organ involvement may be an important factor in terms of disease heterogeneity and LOX, as a component of the pathogenic process leading to fibrotic changes, may reflect such a difference. Yet another reason may underlie the downregulation of LOX expression levels through the progression of skin fibrosis to sclerotic stages. ${ }^{14}$ To the best of our knowledge, the present study is the first to examine the possible relationship between hyperlipidemia and serum LOX concentrations among patients with SSc, and we failed to discover a strong relationship between them. Nevertheless, as all the patients with hyperlipidemia were on lipid-lowering drugs, this outcome should be interpreted with caution. Considering the evidence from the literature, high serum levels of LDL are correlated with the decreased LOX concentrations in human cell cultures. ${ }^{21}$ Moreover, in vitro studies have shown that there is a link between LOX inhibition and an impaired function of the endothelial barrier. ${ }^{21}$ Thus, it is likely that LOX plays a role in the barrier function of the endothelium. As far as the sources of LOX in the serum are concerned, endothelial cells produce LOX. ${ }^{21}$ A more recent study also has suggested connective tissue as another possible source of circulating LOX. ${ }^{28}$ Therefore, some LOX in serum could stem from the endothelium. Accordingly, it can be speculated that not only hyperlipidemia and elevated levels of LDL, but also each condition associated with endothelial dysfunction may influence the ability of endothelial cells to produce LOX and the circulating levels of LOX. In addition, our results indicate that there is a trend toward negative correlation between albumin and LOX serum levels among patients with the diffuse type of the disease, suggesting that there is a possible association between nutritional status in terms of albumin and serum LOX levels. Serum prealbumin concentrations are considered an indication of malnutrition in $\mathrm{SSc}^{29}$ Low levels of prealbumin in the serum have recently been reported as independently predicting mortality in patients suffering from SSc, particularly among those with no internal organ involvement. ${ }^{30}$ Previous studies have also found an association between higher LOX levels in the serum and adverse disease outcomes in SSc. ${ }^{14}$

Nonetheless, there are some limitations to the present study. The main limitation is the poor case selection; we were unable to subdivide the patients according to their inflammatory and molecular signature of the disease. Including patients with similar underlying molecular mechanisms may lead to more accurate results. The second limitation is the small sample size. Yet another limitation regarding dyslipidemia is that we merely evaluated the relationship between the current status of hyperlipidemia and serum LOX levels. Further analysis can focus on the possible correlation of serum LOX levels with the mean concentrations of LDL, high-density lipoprotein, triglyceride, and total cholesterol in the blood. In the present study, all the patients with hyperlipidemia consumed lipid-lowering drugs as well, which may have had a negative effect on the results.

In conclusion, our study results demonstrate that there are significantly higher levels of serum LOX among patients with SSc compared to healthy individuals and that LOX can change over time. Therefore, our findings provide a basis for 
speculations about LOX potentials as a disease status or severity biomarker in SSc. Further largescale studies are required to validate serum LOX levels as a biomarker in SSc. The LOX levels in the blood may change according to the level of disease progression, the years since the first diagnosis, and the age of onset. To elucidate the possible factors influencing LOX levels in the blood, it is also essential to simultaneously examine other conditions associated with endothelial dysfunction.

Ethics Committee Approval: The study protocol was approved by the Institutional Review Board (IRB) Committee of the Golestan University of Medical Sciences (GOUMS) (approval ID: ir.goums.rec.1396.242). The study was conducted in accordance with the principles of the Declaration of Helsinki.

Patient Consent for Publication: A written informed consent was obtained from each participant.

Data Sharing Statement: The data that support the findings of this study are available from the corresponding author upon reasonable request.

Author Contributions: Contributed to the conception, and design of data, draft an article and gave approval to the final version to be published: M.H.J. and S.J.; Contributed to the conception, design, analysis, and interpretation of data, revised it critically for important intellectual content, and gave approval to the final version to be published: N.A. and M.N.; Contributed to the conception, design, and analysis of data, draft an article and gave approval to the final version to be published: M.M. and M.A.M.

Conflict of Interest: The authors declared no conflicts of interest with respect to the authorship and/or publication of this article.

Funding: Golestan University of Medical Sciences financially supported this research (approval ID: ir.goums. rec.1396.242).

\section{REFERENCES}

1. Hämäläinen ER, Jones TA, Sheer D, Taskinen K, Pihlajaniemi T, Kivirikko KI. Molecular cloning of human lysyl oxidase and assignment of the gene to chromosome 5q23.3-31.2. Genomics 1991;11:508-16.

2. Thomassin L, Werneck CC, Broekelmann TJ, Gleyzal $\mathrm{C}$, Hornstra IK, Mecham RP, et al. The Pro-regions of lysyl oxidase and lysyl oxidase-like 1 are required for deposition onto elastic fibers. J Biol Chem 2005;280:42848-55.

3. Csiszar K. Lysyl oxidases: A novel multifunctional amine oxidase family. Prog Nucleic Acid Res Mol Biol 2001;70:1-32.
4. Iftikhar M, Hurtado P, Bais MV, Wigner N, Stephens DN, Gerstenfeld LC, et al. Lysyl oxidase-like-2 (LOXL2) is a major isoform in chondrocytes and is critically required for differentiation. $\mathrm{J}$ Biol Chem 2011;286:909-18.

5. Khosravi R, Sodek KL, Xu WP, Bais MV, Saxena D, Faibish $\mathrm{M}$, et al. A novel function for lysyl oxidase in pluripotent mesenchymal cell proliferation and relevance to inflammation-associated osteopenia. PLoS One 2014;9:e100669.

6. Payne SL, Fogelgren B, Hess AR, Seftor EA, Wiley EL, Fong SF, et al. Lysyl oxidase regulates breast cancer cell migration and adhesion through a hydrogen peroxide-mediated mechanism. Cancer Res 2005;65:11429-36.

7. Bignon M, Pichol-Thievend C, Hardouin J, Malbouyres $\mathrm{M}$, Bréchot $\mathrm{N}$, Nasciutti L, et al. Lysyl oxidase-like protein-2 regulates sprouting angiogenesis and type IV collagen assembly in the endothelial basement membrane. Blood 2011;118:3979-89.

8. Erler JT, Bennewith KL, Nicolau M, Dornhöfer $\mathrm{N}$, Kong $\mathrm{C}$, Le QT, et al. Lysyl oxidase is essential for hypoxia-induced metastasis. Nature 2006;440:1222-6.

9. Chanoki M, Ishii M, Kobayashi H, Fushida H, Yashiro $\mathrm{N}$, Hamada $\mathrm{T}$, et al. Increased expression of lysyl oxidase in skin with scleroderma. $\mathrm{Br} \mathrm{J}$ Dermatol 1995;133:710-5.

10. Nave AH, Mižíková I, Niess G, Steenbock H, Reichenberger F, Talavera ML, et al. Lysyl oxidases play a causal role in vascular remodeling in clinical and experimental pulmonary arterial hypertension. Arterioscler Thromb Vasc Biol 2014;34:1446-58.

11. Meyringer R, Neumann E, Judex M, Landthaler M, Kullmann F, Scholmerich J, et al. Analysis of gene expression patterns in systemic sclerosis fibroblasts using RNA arbitrarily primed-polymerase chain reaction for differential display. J Rheumatol 2007;34:747-53.

12. Jimenez SA. Role of endothelial to mesenchymal transition in the pathogenesis of the vascular alterations in systemic sclerosis. ISRN Rheumatol 2013;2013:835948.

13 Nishimoto T, Takihara T, Mlakar L, Heywood J, Bradshaw A, Feghali-Bostwick C. Lysyl oxidase induces fibrosis via upregulation of IL- 6 and serves as a biomarker to monitor response to therapy. A74 Matrix: Drivers and Targets: American Thoracic Society; 2016. p. A2441-A.

14. Vadasz Z, Balbir Gurman A, Meroni P, Farge D, Levi $Y$, Ingegnoli $F$, et al. Lysyl oxidase-a possible role in systemic sclerosis-associated pulmonary hypertension: A multicentre study. Rheumatology (Oxford) 2019;58:1547-55.

15. Rimar D, Rosner I, Nov Y, Slobodin G, Rozenbaum $\mathrm{M}$, Halasz $\mathrm{K}$, et al. Brief report: Lysyl oxidase is a potential biomarker of fibrosis in systemic sclerosis. Arthritis Rheumatol 2014;66:726-30. 
16. Salter H, Holland R. Biomarkers: Refining diagnosis and expediting drug development - reality, aspiration and the role of open innovation. $J$ Intern Med 2014;276:215-28.

17. Holland RL. What makes a good biomarker? Advances in Precision Medicine 2016;1:4-11.

18. Manno R, Boin F. Immunotherapy of systemic sclerosis. Immunotherapy 2010;2:863-78.

19. Katz R. Biomarkers and surrogate markers: An FDA perspective. NeuroRx 2004;1:189-95.

20. Lafyatis R. Application of biomarkers to clinical trials in systemic sclerosis. Curr Rheumatol Rep 2012;14:47-55.

21. Rodríguez C, Raposo B, Martínez-González J, Casaní L, Badimon L. Low density lipoproteins downregulate lysyl oxidase in vascular endothelial cells and the arterial wall. Arterioscler Thromb Vasc Biol 2002;22:1409-14.

22. Preliminary criteria for the classification of systemic sclerosis (scleroderma). Subcommittee for scleroderma criteria of the American Rheumatism Association Diagnostic and Therapeutic Criteria Committee. Arthritis Rheum 1980;23:581-90.

23. Khanna D, Merkel PA. Outcome measures in systemic sclerosis: An update on instruments and current research. Curr Rheumatol Rep 2007;9:151-7.

24. Giampuzzi M, Botti G, Di Duca M, Arata L, Ghiggeri $\mathrm{G}$, Gusmano R, et al. Lysyl oxidase activates the transcription activity of human collagene III promoter. Possible involvement of $\mathrm{Ku}$ antigen. $\mathrm{J}$ Biol Chem 2000;275:36341-9.

25. Storey JD, Madeoy J, Strout JL, Wurfel M, Ronald J, Akey JM. Gene-expression variation within and among human populations. Am J Hum Genet 2007;80:502-9.

26. Gonzàlez-Porta M, Calvo M, Sammeth M, Guigó R. Estimation of alternative splicing variability in human populations. Genome Res 2012;22:528-38.

27. de Bruyn JR, Meijer SL, Wildenberg ME, Bemelman WA, van den Brink GR, D'Haens GR. Development of fibrosis in acute and longstanding ulcerative colitis. J Crohns Colitis 2015;9:966-72.

28. Nguyen XX, Nishimoto T, Takihara T, Mlakar L, Bradshaw AD, Feghali-Bostwick C. Lysyl oxidase directly contributes to extracellular matrix production and fibrosis in systemic sclerosis. Am J Physiol Lung Cell Mol Physiol 2021;320:L29-L40.

29. Caporali R, Caccialanza R, Bonino C, Klersy C, Cereda E, Xoxi B, et al. Disease-related malnutrition in outpatients with systemic sclerosis. Clin Nutr 2012;31:666-71.

30. Codullo V, Cereda E, Klersy C, Cavazzana I, Alpini C, Bonardi C, et al. Serum prealbumin is an independent predictor of mortality in systemic sclerosis outpatients. Rheumatology (Oxford) 2016;55:315-9. 